\title{
Enteroaggregative Escherichia coli expresses a novel flagellin that causes IL-8 release from intestinal epithelial cells
}

\author{
Ted S. Steiner, ${ }^{1}$ James P. Nataro, ${ }^{2}$ Celeste E. Poteet-Smith, ${ }^{3}$ \\ Jeffrey A. Smith, ${ }^{3}$ and Richard L. Guerrant ${ }^{1}$ \\ ${ }^{1}$ Division of Geographic and International Medicine, University of Virginia Health Sciences Center, Charlottesville, \\ Virginia, USA \\ ${ }^{2}$ Center for Vaccine Development, University of Maryland, Baltimore, Maryland, USA \\ ${ }^{3}$ Howard Hughes Medical Institute and the Markey Center for Cell Signaling, Department of Medicine and Pharmacology, \\ University of Virginia Health Sciences Center, Charlottesville, Virginia, USA
}

Address correspondence to: Ted Steiner, Box 801379 HSC, Charlottesville, Virginia 22908, USA. Phone: (804) 924-5242; Fax: (804) 977-5323; E-mail: ts5x@virginia.edu.

Received for publication November 11, 1999, and accepted in revised form May 2, 2000.

\begin{abstract}
Enteroaggregative Escherichia coli (EAEC) is an emerging cause of acute and persistent diarrhea worldwide. EAEC infections are associated with intestinal inflammation and growth impairment in infected children, even in the absence of diarrhea. We previously reported that prototype EAEC strains rapidly induce IL-8 production by Caco- 2 intestinal epithelial cells, and that this effect is mediated by a soluble, heat-stable factor released by these bacteria in culture. We herein report the cloning, sequencing, and expression of this biologically active IL-8-releasing factor from EAEC, and its identification as a flagellin that is unique among known expressed proteins. Flagella purified from EAEC 042 and several other EAEC isolates potently release IL-8 from Caco-2 cells; an engineered aflagellar mutant of 042 does not release IL-8. Finally, cloned EAEC flagellin expressed in nonpathogenic E. coli as a polyhistidine-tagged fusion protein maintains its proinflammatory activity. These findings demonstrate a major new means by which EAEC may cause intestinal inflammation, persistent diarrhea, and growth impairment that characterize human infection with these organisms. Furthermore, they open new approaches for diagnosis and vaccine development. This novel pathogenic mechanism of EAEC extends an emerging paradigm of bacterial flagella as inflammatory stimuli.
\end{abstract}

J. Clin. Invest. 105:1769-1777 (2000).

\section{Introduction}

The term enteroaggregative Escherichia coli (EAEC) refers to a heterogeneous group of bacteria defined by a phenotypic assay, aggregative (or "stacked-brick") adherence to HEp-2 cells in culture. Since it was first described in 1987, EAEC has emerged as a leading cause of persistent childhood diarrhea in many developing areas $(1,2)$. More recently, EAEC has been identified in developed countries, causing acute, persistent, or chronic diarrhea in travelers $(3,4)$, patients with AIDS (5), and children attending day-care centers (6), and appearing in large food-borne outbreaks (7). In addition to causing diarrhea, pediatric EAEC infections are associated with growth shortfalls and intestinal inflammation, even in children without diarrhea (8). In this setting, we recently documented that early childhood diarrhea correlates with long-term deficits in growth, physical fitness, and cognitive function (9). The long-term effects of EAEC infection on growth and development of children in developing areas may be even more important than the short-term morbidity associated with diarrheal illness.

Based on our clinical studies showing evidence of intestinal inflammation with EAEC infections in children in Brazil, we have reported proinflammatory activity of EAEC culture supernatants, mediated by a putative protein that causes rapid release of the neutrophil chemokine IL-8 from Caco- 2 human colonic carcinoma cells (8). Herein we report the purification, cloning, expression, and identification of this protein as a flagellin unique among known E. coli flagellar proteins, but homologous to an unexpressed flagellin from Shigella dysenteriae. This finding generates a novel paradigm of E. coli enteric pathogenesis.

\section{Methods}

Cell culture and $I L-8$ release. Cell culture reagents were obtained from GIBCO BRL (Bethesda, Maryland, USA) unless otherwise specified. Caco-2 human colonic carcinoma cells were obtained from Cynthia Sears (Johns Hopkins University, Baltimore, Maryland, USA), and were grown as described (8). CCD-18Co human colonic fibroblasts were obtained from American Type Culture Collection (Rockville, Maryland, USA), and were grown in MEM with $2 \mathrm{mM}$ L-glutamine, $1.5 \mathrm{~g} / \mathrm{L} \mathrm{NaHCO}_{3}, 1$ mM sodium pyruvate, $0.1 \mathrm{mM}$ nonessential amino acid solution, $100 \mathrm{U} / \mathrm{mL}$ penicillin, $100 \mu \mathrm{g} / \mathrm{mL}$ streptomycin sulfate, and 10\% FBS. Fibroblasts were serially passaged using $0.25 \%$ trypsin and $1 \mathrm{mM}$ EDTA. T84 
human colonic carcinoma cells were obtained from American Type Culture Collection and were grown in 50\% DMEM and 50\% Ham's F12 supplemented with $15 \mathrm{mM}$ HEPES, $26 \mathrm{mM} \mathrm{NaHCO}_{3}$, 5\% FBS, $100 \mathrm{U} / \mathrm{mL}$ penicillin, and $100 \mu \mathrm{g} / \mathrm{mL}$ streptomycin sulfate; these were passaged using 1\% trypsin/ 1 mM EDTA.

For IL-8 release assays, cells seeded at a density of 500,000 per well into 24-well polystyrene plates were used after 5-14 days. Bacteria or bacterial products to be tested were added to $500 \mu \mathrm{L}$ of growth medium in each well. After 3 hours of incubation at $37^{\circ} \mathrm{C}$ in an atmosphere of $5 \% \mathrm{CO}_{2}$, the medium was removed and tested for IL-8 concentration by enzyme immunoassay (Quantikine IL-8; R\&D Systems, Minneapolis, Minnesota, USA). One unit of activity was defined as the amount of protein that produced half-maximal IL-8 release. When live bacteria were used, the Caco- 2 cells were rinsed thoroughly, and the media was replaced with an identical media lacking penicillin and streptomycin before bacteria were added. After 3 hours, supernatants were removed. Cell monolayers were fixed with methanol for 5 minutes and then stained with Giemsa stain for 20 minutes to visualize bacteria and biofilm.

Bacterial preparations. The bacterial isolates used in this study are described in Table 1. For Caco-2 IL-8 release assays, bacteria were grown for 16-24 hours in $1 \%$ tryptone at $37^{\circ} \mathrm{C}$ with shaking at $200-300 \mathrm{rpm}$. Either $20 \mu \mathrm{L}$ of live bacterial culture or $50 \mu \mathrm{L}$ of $0.2 \mu \mathrm{m}$ filtered culture supernatant was added to the Caco-2 cells, and the mixture was incubated for 3 hours.

To isolate flagella, bacteria were pelleted by centrifugation, resuspended in $30 \mathrm{~mL}$ of $500 \mathrm{mM}$ Tris $(\mathrm{pH}$ 8.0), and then blended at high speed for 60 seconds in a Sorvall Omni Mixer (Sorvall Products, Newtown, Connecticut, USA). Bacteria and debris were pelleted by centrifugation at $8,000 \mathrm{~g}$ for 15 minutes, and the supernatant was clarified by filtration $(0.8 \mu \mathrm{m}$ pore size). Flagella were pelleted by centrifugation at $100,000 \mathrm{~g}$ for 60-90 minutes and then resuspended in $5 \mathrm{~mL}$ of PBS. For smaller culture volumes, flagellin was isolated by acid denaturation. Bacterial pellets were suspended in a small volume of $150 \mathrm{mM} \mathrm{NaCl}$ and $10 \mathrm{mM} \mathrm{HCl}$, and rotated for 30 minutes at room temperature. Supernatant containing depolymerized flagella was clarified by centrifugation at $100,000 \mathrm{~g}$ for 60-90 minutes and equilibrated to $\mathrm{pH} 8.0$ by addition of $50 \mathrm{mM}$ Tris and $10 \mathrm{mM} \mathrm{NaOH}$. The apparent molecular weight of flagellin on SDS-PAGE was the same regardless of the method used.

Protein purification techniques. The IL-8-releasing protein was purified from culture supernatants of EAEC 042, the prototype strain of EAEC that was diarrheagenic in volunteers (10). All purification steps were performed at $4{ }^{\circ} \mathrm{C}$. Ultrafiltration membranes and concentrators (Amicon YM-10 membrane, Centricon-30, and Centriplus-30) were obtained from Millipore Corp. (Bedford, Massachusetts, USA). Chromatography was performed using the Gradi-Frac and FPLC systems from Pharmacia Biotech AB (Uppsala, Sweden). The following chromatographic supports and columns were used: DEAE-cellulose (Sigma Chemical Co., St. Louis, Missouri, USA); Sephacryl S-300 16/60, MonoQ HR 5/5, Butyl-Sepharose fast flow (Pharmacia Biotech AB); and Bio-Gel HTP hydroxyapatite (Bio-Rad Laboratories Inc., Hercules, California, USA). Protein concentration was measured by the Bradford method (using protein assay reagent from Bio-Rad Laboratories Inc.). Proteins were visualized by electrophoresis through $9 \%$ polyacrylamide-SDS gels by the Laemmli method and staining with Coomassie blue, Gelcode Blue (Pierce Chemical Co., Rockford, Illinois, USA), or ammoniacal silver nitrate.

Protein sequencing. After sequential ion exchange, gel permeation, hydrophobic interaction, and hydroxyapatite column chromatography, IL-8-releasing material was concentrated by acetone precipitation and run on a $9 \%$ polyacrylamide-SDS gel. The single visible band of approximately $65 \mathrm{kDa}$ was digested in situ with trypsin and sequenced by tandem mass spectrometry at the W.M. Keck Biomedical Mass Spectrometry Laboratory at the University of Virginia. Homology to published peptide sequences was determined using the BLAST program from the National Center for Biotechnology Information.

Production of aflagellar mutant. The following primers were engineered from the middle third of the $f l i C_{\mathrm{SD}}$ sequence (from nucleotides encoding peptides matching two of those obtained from the EAEC flagellin during mass spectrometry) with $X b a I$ and EcoRI sites, respectively, at the $5^{\prime}$ ends: 5'-TCTAGATAAAACTCGATAATACGGGGG-3' and 5'-GAATTCTAACGGCGTTACGAAACGTTGT-3'. The 588-bp product amplified with these primers from 042 genomic DNA (using Taq polymerase from QIAGEN Inc., Valencia, California, USA) was ligated into the R6K suicide plasmid vector PJP5603, which replicates only when the pir gene product is supplied in trans (11), and electroporated into DH5 $\alpha(\lambda$ pir $)$. Transformants were selected by growth on Luria broth (LB) agar with kanamycin $(30 \mu \mathrm{g} / \mathrm{mL})$, and the correct plasmid was identified by restriction pattern. This plasmid was then electroporated into the conjugative strain $\mathrm{S} 17-1(\lambda$ pir), and was conjugated into 042 by filter mating. Transconjugants, predicted to arise by chromosomal insertion of the vector by homologous recombination, were selected by growth on LB with kanamycin $(30 \mu \mathrm{g} / \mathrm{mL})$ and tetracycline $(24$ $\mu \mathrm{g} / \mathrm{mL}$ ), and then screened for IL-8-releasing activity. Southern blotting was performed on Zeta-Probe GT membranes (Bio-Rad Laboratories Inc.) according to the manufacturer's protocol. Motility was assessed by growth on swarm plates ( $1.55 \mathrm{~g} / \mathrm{L} \mathrm{LB}$ and $0.3 \%$ agar). HEp-2 adherence was determined as in ref. 2.

Plasmid rescue from transconjugants was performed as follows: Genomic DNA was cut with BamHI and ligated in dilute solution to facilitate homotopic ligation. Ligation product was electroporated into DH5 $\alpha(\lambda$ pir $)$ and plated on kanamycin agar. Colonies were screened by hybridization with 
Table 1

Bacterial isolates used in this study

\begin{tabular}{|c|c|c|c|c|}
\hline & Source & Serotype & Mean 3-hour IL-8 release from Caco-2 cells by flagella $(\mathrm{pg} / \mathrm{mL})^{\mathrm{A}}$ & $n$ \\
\hline \multicolumn{5}{|l|}{ EAEC isolates } \\
\hline 042 & Peru & 044:H18 & 564.6 & 4 \\
\hline 1096 & Serbia & $\mathrm{O} 4: \mathrm{H}$ ? & 322.5 & 2 \\
\hline W452 & Thailand ${ }^{B}$ & $\mathrm{O} ?: \mathrm{H} 18$ & 454.6 & 2 \\
\hline W44 & Thailand $^{B}$ & $\mathrm{O} 36: \mathrm{H} 18$ & $<20$ & 2 \\
\hline W202 & Thailand ${ }^{B}$ & $\mathrm{O}: \mathrm{H} 18$ & 393.6 & 2 \\
\hline GDI 44 & c & D & $<20$ & 2 \\
\hline GDI 180 & c & D & 86.5 & 2 \\
\hline GDI 252 & c & D & 196.25 & 1 \\
\hline GDI 299 & c & $\mathrm{O}$ ?:H18 & 450.6 & 2 \\
\hline $17-2$ & Chile & ND & 30.3 & 1 \\
\hline \multicolumn{5}{|c|}{ Other pathogenic isolates } \\
\hline V. cholerae (JBK70) & E & - & $<20$ & 2 \\
\hline E2348 (EPEC) & CVD (ref. 30) & O127:H6 & 283.6 & 3 \\
\hline 933L (EHEC) & $\mathrm{F}_{\mathrm{F}}$ & O157:H7 & 573.7 & 2 \\
\hline C1845 (DAEC) & CVD (ref. 30) & ND & $<20$ & 2 \\
\hline \multicolumn{5}{|c|}{ Nonpathogenic isolates } \\
\hline GDI 20 & c & D & $<20$ & 3 \\
\hline GDI 16 & c & D & 190.8 & 2 \\
\hline BL21(DE3)pLysS & Invitrogen Corp. & ND & $<20$ & 1 \\
\hline Top10F' & Invitrogen Corp. & ND & ND & \\
\hline $\mathrm{DH} 5 \alpha(\lambda$-pir $)$ & CVD & ND & ND & \\
\hline $\mathrm{S} 17-1(\lambda$-pir $)$ & CVD & ND & ND & \\
\hline HB101 & CVD & ND & ND & \\
\hline
\end{tabular}

AFlagella prepared by acid depolymerization were added to Caco-2 cells at $0.5-5.0 \mu \mathrm{g} /$ well and supernatant was assayed for IL-8 by ELISA. ${ }^{\mathrm{B}}$ Obtained from Peter Echeverria (Armed Forces Research Institute of Medical Sciences, Bangkok, Thailand); serotyped by Hermy Lior (Laboratory Centre for Disease Control, Ottawa, Ontario, Canada). 'Conçalves Dias favela, Fortaleza, Brazil. DNot serotyped, but negative for slide agglutination with H18 antiserum. EV. cholerae expressing only B subunit of cholera toxin; obtained from James Kaper (Center for Vaccine Development, University of Maryland; ref. 29). FSLT-I-positive, SLTII-negative strain, obtained from Alison O'Brien (Uniformed Services University of the Health Sciences, Bethesda, Maryland, USA). ND, not done; EPEC, enteropathogenic E. coli; EHEC, enterohemorrhagic E. coli; DAEC, diffusely adherent $E$. coli.

the 588-bp probe described above, and the resulting plasmid $(\sim 15 \mathrm{~kb})$ was sequenced using the pUC19 forward and reverse primers.

Electron microscopy. Bacteria from saturated overnight culture were pelleted by gentle centrifugation and then resuspended in filtered distilled water. Aliquots $(10 \mu \mathrm{L})$ of the suspension were applied to carbon-coated formvar grids for approximately 3-4 minutes. After a quick blotting of the excess suspension, $10 \mu \mathrm{L}$ of ammonium molybdate was applied for $5-15$ seconds. The stain was then blotted off, and the grids were allowed to air dry. The bacteria were observed and photographed in a JEOL 100CX (JEOL USA Inc., Peabody, Massachusetts, USA) electron microscope at $80 \mathrm{kV}$.

Cloning and expression of the EAEC flagellin. The following primers were designed from the published $\mathrm{NH}_{2}$ - and $\mathrm{COOH}$-terminal sequences of the $\mathrm{S}$. dysenteriae flagellin: 5'-GGATTCATGGCACAAGTCATTAAT-3' and 5'-TTCGAATTAACCCTGCTGCAGAGA-3'. These were used to amplify a fragment of approximately 1.8 $\mathrm{kb}$ from genomic DNA isolated from EAEC 042. This fragment was cloned in frame with a $5^{\prime}$ six-histidine (six-His) tag in the pCRT7/NT-TOPO vector (Invitrogen Corp., Carlsbad, California, USA) and maintained in a Top $10 \mathrm{~F}^{\prime}$ host, according to the manufacturer's protocol. The plasmid containing the fliC gene in frame $\mathrm{pfliC}$ was verified by sequencing, and the fusion protein was expressed in BL21(DE3)pLysS according to the manufacturer's protocol. The control plasmid provided by the manufacturer (pCRT7/NT-E3), expressing a 58-kDa human kinase, was expressed in parallel as a control. Cells were harvested by centrifugation two hours after induction of mid-log phase culture with $1 \mathrm{mM}$ isopropyl $\beta$-Dthiogalactopyranoside (IPTG). Lysis was performed in the manufacturer's recommended lysis buffer by repeated freeze-thaw and sonication. His-tagged proteins were loaded on $\mathrm{NiCl}_{2}$-charged 1 -mL metal chelate columns (Pharmacia Biotech $\mathrm{AB}$ ) in a buffer containing $500 \mathrm{mM} \mathrm{NaCl}, 20 \mathrm{mM}$ Tris ( $\mathrm{pH} 8.0$ ), and $5 \mathrm{mM}$ imidazole. They were then washed with $20 \mathrm{mM}$ imidazole and eluted in similar buffer with $100 \mathrm{mM}$ imidazole. The flagellin fusion protein was identified by ELISA using $\mathrm{Ni}^{2+}$-conjugated horseradish peroxidase according to the manufacturer's protocol (India HisProbe-HRP; Pierce Chemical Co.) and by Western blot (see below).

Western blotting. Proteins were run on 9\% SDS-PAGE gels and electrotransferred to PVDF membranes (Millipore Corp.) at $30 \mathrm{~V}$ overnight. They were blocked using $5 \%$ nonfat dry milk in TTBS $(20 \mathrm{mM}$ Tris at $\mathrm{pH}$ $7.5,150 \mathrm{mM} \mathrm{NaCl}$, and $0.05 \%$ Tween-20), and then hybridized for 2 hours with Salmonella $\mathrm{H}$ antiserum poly a-z (Difco Laboratories, Detroit, Michigan, USA) at a dilution of $1: 1,000$ in TTBS with $1 \%$ gelatin. This was followed by 1 hour with peroxidase-conjugated donkey anti-rabbit IgG (Amersham Life Science, Buckinghamshire, United Kingdom) and developing with ECL light detection reagents (Amersham Life Science). His-tagged proteins were identified by hybridization with India HisProbe-HRP according to the manufacturer's protocol. 


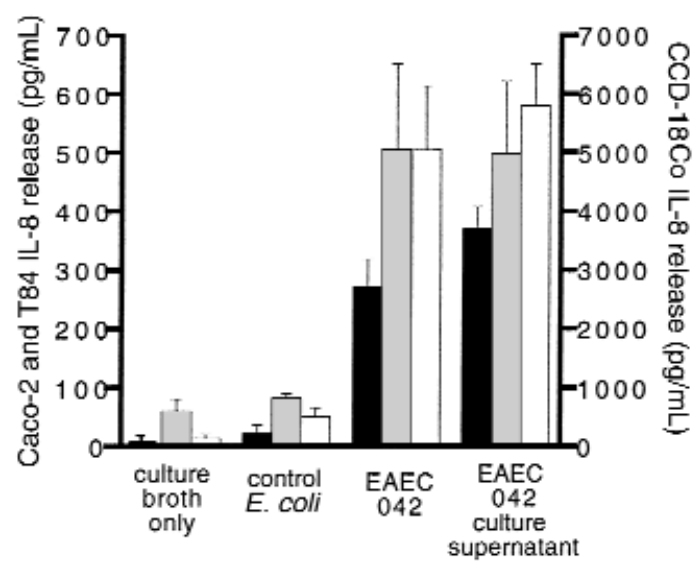

Figure 1

IL-8 release from intestinal cell lines in response to EAEC. Five-dayold monolayers of Caco-2 (black bars), T84 (gray bars), or CCD18Co (white bars) cells were exposed for 3 hours to live EAEC 042, filtrates of saturated overnight cultures of EAEC 042, nonpathogenic (control) E. coli (HB101, K12, or GDI 20), or culture broth alone. The separate $Y$-axes reflect the tenfold greater amount of IL-8 release by CCD-18Co fibroblasts compared with Caco- 2 or T84 colonic carcinoma cells. Error bars represent SEM.

\begin{abstract}
Results
EAEC 042 induces IL-8 release from intestinal cell lines. We previously reported that a prototype EAEC isolate, 042 , causes Caco- 2 cells to release IL-8 after as little as 3 hours of exposure to either live bacteria or to cell-free culture filtrates. We also demonstrated the heat stability and protease sensitivity of this IL-8-releasing activity, and its lack of inhibition by polymyxin B (8). Because proinflammatory cytokines are regulated differently in various immortalized cell lines, we tested T84 human colonic carcinoma cells and CCD-18Co human colonic fibroblasts. As shown in Figure 1, EAEC 042, the prototype strain that is pathogenic in volunteers, caused significantly more IL-8 release than did nonpathogenic E. coli or culture broth alone in all three cell types. Interestingly, T84 and CCD-18Co cells both express measurable IL-8 at baseline, whereas Caco-2 cells do not. For this reason, we used Caco-2 cells as the primary assay cells for the IL-8-releasing protein.

Purification and identification of the IL-8-releasing protein from EAEC 042. We purified the IL-8-releasing protein from culture supernatants of EAEC 042. The appearance of the sequential purification products on $9 \%$ SDS-PAGE gels is shown in Figure 2.
\end{abstract}

The SDS-PAGE band from the hydroxyapatite chromatography step was sequenced by tandem mass spectroscopy. Eighteen trypsin peptides were identified that matched predicted sequences from a presumed $S$. dysenteriae flagellin, fli $\mathrm{C}_{\mathrm{SD}}$ (National Center for Biotechnology Information accession 442483). Eleven of these peptides were identical to the corresponding sequences predicted from $f \mathrm{C}_{\mathrm{SD}}$; the remainder had single amino-acid substitutions. None of the identified peptides matched predicted peptide sequences of the flagellin from $\mathrm{E}$. coli K-12 (fliC gene product, accession 120319).

Flagella from EAEC cause IL-8 release from Caco-2 cells. To verify that the identified flagellin was indeed the IL-8-releasing protein from 042, we prepared flagella from EAEC 042 (Figure 3). We cut the higher- and lower-molecular-weight bands from a gel, eluted the proteins into water by diffusion, and dialyzed them thoroughly to remove SDS. The smaller proteins did not cause IL-8 release, whereas the protein of about $65 \mathrm{kDa}$ (henceforth referred to as FliC-EAEC) was extremely potent, with a specific activity of approximately $333 \mathrm{U} / \mu \mathrm{g}$, corresponding to half-maximal activity at approximately $10^{-10} \mathrm{M}$. Moreover, when EAEC flagella were treated at $\mathrm{pH} 3.0$ and subsequently neutralized, they were still active and maintained their mobility and staining characteristics on SDS-PAGE (not shown). This suggests that depolymerized flagella (consisting largely of the flagellin protein) are as active as intact flagella, and hence that the flagellin itself is responsible for IL-8 release. As with EAEC culture supernatants, addition of polymyxin B $(10 \mu \mathrm{g} / \mathrm{mL})$ to Caco-2 cells did not reduce the IL-8-releasing effect of EAEC flagellin, suggesting that LPS did not contribute to this effect.

Several additional strains of bacteria were tested for the presence of IL-8-releasing flagella (Table 1). No detectable IL- 8 release was induced in Caco- 2 cells by the addition of $50 \mu \mathrm{L}$ of flagellar solution (0.5-5.0 $\mu \mathrm{g} /$ well) from either GDI 20 (a nonpathogenic human isolate from Brazil), Vibrio cholerae, C1845 (a diffusely adherent $E$. coli), or BL21. In contrast, proinflammatory flagella were detected in eight of ten EAEC isolates from diverse sources, as well as enterohemorrhagic E. coli O157:H7 and enteropathogenic E. coli E2348. Interestingly, there was a dissociation between reactivity with $\mathrm{H} 18$ antiserum and IL-8

\section{Figure 2}

Gel (9\% SDS-PAGE) of sequentially purified, IL-8-releasing protein fractions from EAEC 042 culture supernatants. Samples were concentrated by membrane ultrafiltration to a volume of $25 \mu \mathrm{L}$ and run simultaneously, with the exception of lane 7 , which was run in a separate gel. Lane 1: Culture supernatant from overnight growth of 042. Lane 2: DEAE-cellulose batch adsorption and elution with $\mathrm{NaCl}$. Lane 3: Sixty percent ammonium sulfate precipitation. Lane 4: Gel permeation chromatography in the presence of $6 \mathrm{M}$ guanidine- $\mathrm{HCl}$. Lane 5: Pooled, active gel permeation fractions, dialyzed to remove guanidine. Lane 6: Strong anion exchange and subsequent hydrophobic interaction chromatography. Lane 7: Hydroxyapatite chromatography.

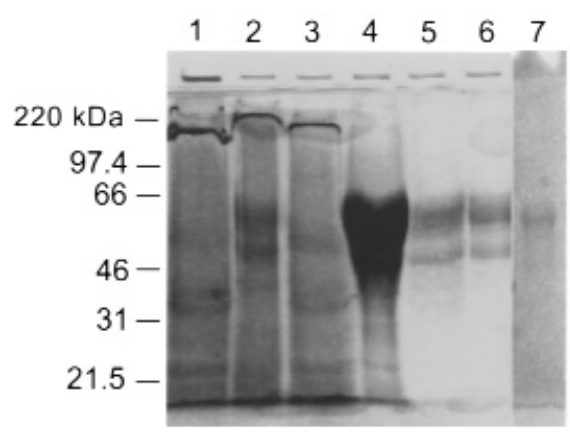




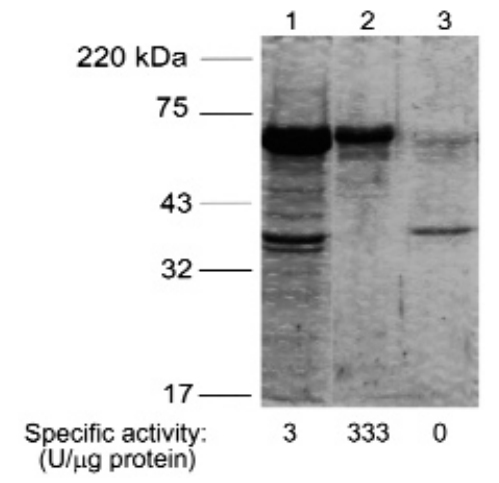

\section{Figure 3}

Gel (9\% SDS-PAGE) of EAEC flagellar isolation. Samples were concentrated by acetone precipitation before loading. Lane 1: Flagella sheared from EAEC 042 and pelleted by ultracentrifugation. Lane 2: The approximately $65-\mathrm{kDa}$ band, cut and eluted from a previous gel, dialyzed to remove SDS, and reVrun in SDS-containing loading buffer. Lane 3: The approximately 35-kDa band similarly eluted, dialyzed, and rerun. Specific activity was defined as the amount of sample producing half-maximal IL-8 release from Caco-2 cells at 3 hours of exposure.

release, suggesting that the $\mathrm{H}$-antigenic epitopes on FliC-EAEC may be separate from the regions of the molecule responsible for proinflammatory activity. Aflagellar EAEC 042 does not cause IL-8 release. To determine if flagellar expression is necessary for 042 to cause IL-8 release, we used a 588-bp internal fragment of fliC to direct insertion of the R6K suicide vector pJP5603 into the EAEC 042 flagellin gene $\left(f l i C_{\mathrm{EAEC}}\right)$. This strategy produced a partial merodiploid, 042: $f l i \mathrm{C}^{-}$, harboring the suicide vector (Figure 4a). The identity of $042: \mathrm{fliC}^{-}$as a derivative of 042 was confirmed by antibiotic susceptibility pattern (resistant to chloramphenicol and tetracycline), by aggregative adherence to HEp-2 cells, by biofilm formation, by the presence of a plasmid with the same size and restriction patterns as the $042 \mathrm{AA}$ plasmid, and by agglutination with O44 antiserum (Denka Seiken Co., Tokyo, Japan). Southern hybridization confirmed the site of insertion of the suicide plasmid (Figure 4b). Moreover, plasmid rescue liberated a novel plasmid of approximately $15 \mathrm{~kb}$ harboring the R6K origin and kanamycin resistance cassette.

\section{Figure 4}

Construction of 042:flic- (a) Restriction maps of pJP5603 (3.1 kb) with the cloned 588-bp PCR fragment of fli $C_{\text {EAEC }}$ (gray bar), wild-type fli $C_{\mathrm{EAEC}}$ (dashed line), and the map of the desired transconjugant. Restriction sites and locations are shown (Bam, BamHI; Ssp, Sspl; Stu, Stul; Spe, Spel; Xba, Xbal; Eco, EcoRI). (b) Southern hybridization of 042 and 042:flic- using the 588-bp PCR fragment from the 042 genome as a probe. Lane 1: Probe, $1 \mathrm{ng}$. Lane 2: 042, Spel/Stul cut. Lane 3: 042, Spel/Sspl cut. Lane 4: 042:flic-, Spel/Stul cut. Lane 5: 042:flic-, Spel/Sspl cut. Kmr, kanamycin resistance cassette; TTA, stop codon; ori R6K, origin of replication.
Sequencing of the portions of this plasmid flanking the pJP5603/fliC junction revealed the predicted duplicated copy of the 588-bp region of the fliC gene, confirming the partial merodiploid (not shown).

IL-8-releasing activities of 042 and $042: \mathrm{fliC}^{-}$were compared using both live bacteria and culture supernatants. As shown in Figure 5c, neither live 042:fliC- $(n=3)$ nor its culture supernatants $(n=5)$ caused detectable IL-8 release from Caco-2 cells at similar bacterial density to that from active cultures of 042 (as determined by dilutional colony counts of the bacterial cultures).

042:fli $\mathrm{C}^{-}$demonstrated reduced motility compared with wild-type 042 when grown on swarm plates (Figure 5a). Other phenotypic characteristics of 042 (growth, adherence, biofilm formation) were not altered in 042:fli $\mathrm{C}^{-}$. Reversion to a motile state was able to occur spontaneously in the absence of kanamycin selection, presumably by a recombination event facilitated by the repeated portion of the fliC gene. Reversion to wild-type motility also restored IL-8-releasing activity (not shown).

Several strains were examined for flagella by electron microscopy. As shown in Figure 5b, 042 is highly flagellated, whereas 042:fli $\mathrm{C}^{-}$has no visible flagella (over many fields). In addition, 042 grown in LB with $3 \%$ $\mathrm{NaCl}$ had greatly reduced flagellar expression and did not release IL-8 from Caco-2 cells; adding $3 \% \mathrm{NaCl}$ after overnight growth had no effect on IL-8 release.

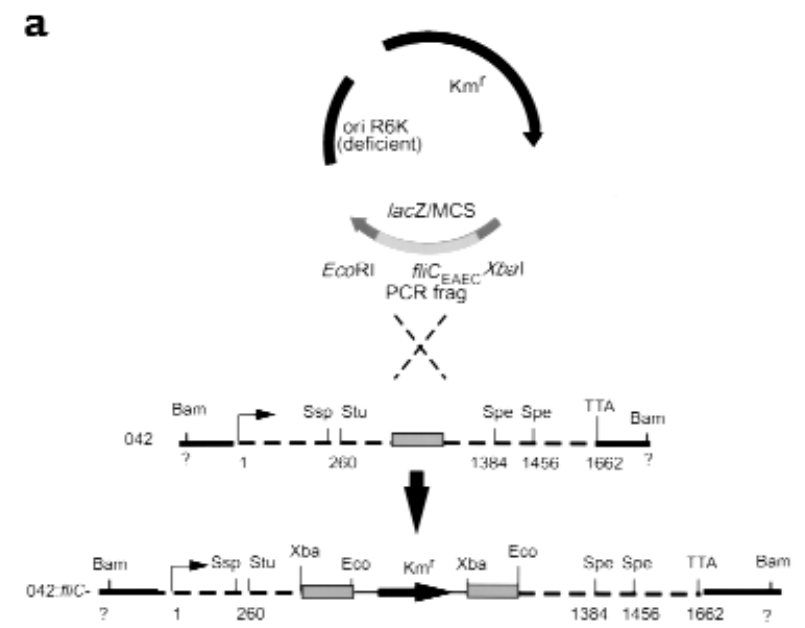

b

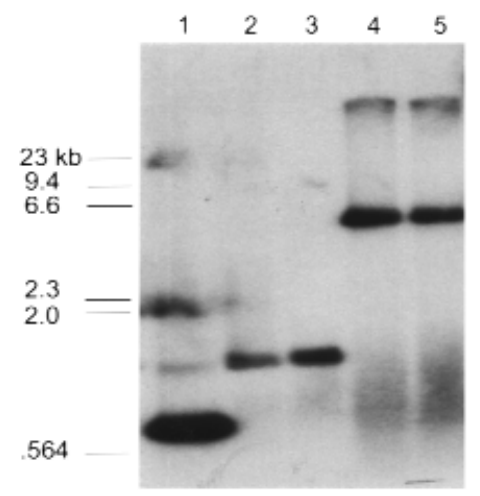


a

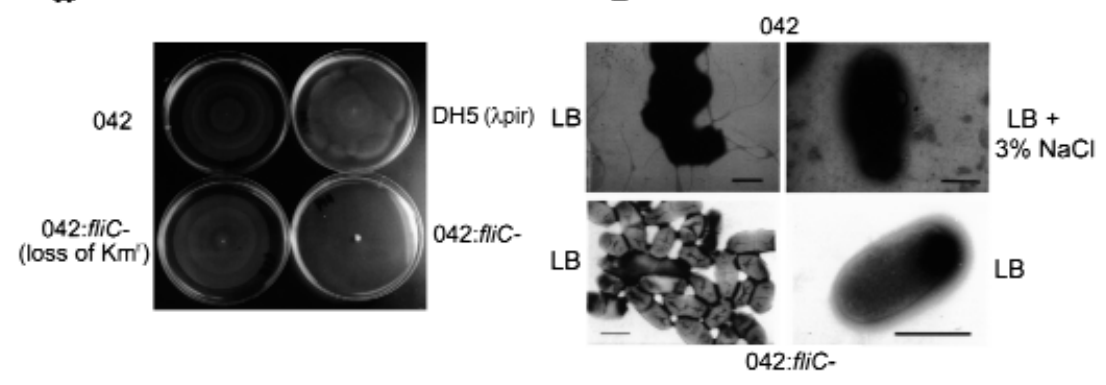

C

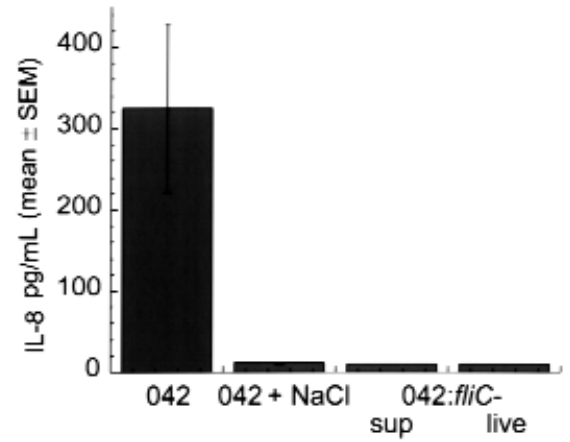

Figure 5

Motility and IL-8-releasing activity of 042 and 042:flic- (a) Growth at 72 hours on swarm plates without kanamycin. Bacteria picked from the periphery (swarming area) of the lower left plate had lost kanamycin resistance. (b) Electron microscopy with negative staining for flagella. Bar $=$ $1 \mu \mathrm{m}$. (c) IL-8 release from Caco-2 cells incubated for 3 hours with culture supernatants of EAEC 042 grown in $1 \%$ tryptone $(n=5), 1 \%$ tryptone with $3 \% \mathrm{NaCl}(n=2)$, culture supernatants (sup) $(n=5)$, or live cultures $(n=3)$ of $042: f l C^{-}$grown in $1 \%$ tryptone. $P<0.05$ by Kruskal-Wallis test.

EAEC flagellin expressed in BL21 causes $I L-8$ release. As described in Methods, the fliC gene was cloned into an expression vector with an $\mathrm{NH}_{2}$-terminal 6-His tag. Maximal expression occurred 2 hours after addition of IPTG to mid-log phase cultures, although some expression was present in unstimulated cultures, reflecting basal transcription at the T7 promoter. As shown in Figure 6, FliC-EAEC, but not the expressed control protein, immunoreacted with anti-flagellar antibody, whereas both proteins reacted with $\mathrm{Ni}^{2+}$ HRP. Crude lysates of BL21-pLysS:pfliC, but not BL21-pLysS:pCRT7/NT-E3, caused IL-8 release from Caco- 2 cells. The IL-8-releasing specific activity of lysates was increased by purification over a $\mathrm{Ni}^{2+}$ column (not shown).

H18 antiserum inhibits IL-8 release by the 042 flagellin. As mentioned earlier, EAEC 042 has a serotype of O44:H18. We used commercially available antiserum to the H18 flagellum (Denka Seiken Co.) to attempt

\section{Figure 6}

Expression and purification of FliC-EAEC. (a) BL21(DE3)pLysS transformed with pfliC or PCRT7/NT-E3 (expression control) were harvested from mid-log phase cultures at the indicated times after addition of IPTG. Ten microliters of bacterial lysate was loaded per well for $9 \%$ SDS-PAGE, and transferred to PVDF membrane after gel was run. The membrane was probed with $\mathrm{Ni}^{2+}-\mathrm{HRP}$ to detect polyhistidine-containing proteins, stripped with $0.2 \mathrm{~N} \mathrm{NaOH}$, and reprobed with polyclonal anti-flagellar antiserum. A lane containing $2.5 \mu \mathrm{g}$ of EAEC 042 flagella $\left({ }^{A}\right)$ was included in the gel as a control. For IL-8 release, purified flagella $(72 \mathrm{ng})$ or bacterial lysate $(1 \mu \mathrm{g})$ was added to $500-\mu \mathrm{L}$ wells of Caco- 2 cells in the same experiment, and supernatant was assayed after 3 hours of incubation. (b) BL21(DE3)pLysS:pfliC was harvested 2 hours after addition of IPTG to mid-log phase culture. Lysates were purified by nickel affinity chromatography, visualized on 9\% SDS-PAGE gels, and transferred to a PVDF membrane that was probed with $\mathrm{Ni}^{2+}$-HRP. Lane 1: EAEC 042 flagella, $1.4 \mu \mathrm{g}$. Lane 2: Crude lysate, $15 \mu \mathrm{g}$. Lane 3: Flowthrough from nickel column, $12.5 \mu \mathrm{g}$. Lane 4: Eluate from column, $10.8 \mu \mathrm{g}$. For IL-8 release, $350 \mathrm{ng}$ of flagella, $1 \mu \mathrm{g}$ of bacterial lysate, and $1 \mu \mathrm{g}$ of eluted flagellin were tested. to adsorb FliC-EAEC and inhibit IL-8 release from Caco- 2 cells. This antiserum immunoreacts by Western blot with flagellin purified from 042 or FliCEAEC expressed in BL21 (not shown). Addition of $1-10 \mu \mathrm{L}$ of $\mathrm{H} 18$ antiserum to Caco- 2 cells inhibited IL-8 release from Caco- 2 cells stimulated with 042 culture supernatants, purified flagella, or His-tagged FliC-EAEC to $51.2 \pm 11.2 \%$ of control levels (mean \pm $\mathrm{SD}, n=4, P=0.025)$. No inhibition of IL-8 release was seen with $\mathrm{H} 7$ antiserum or polyclonal anti-Salmonella a-z antiserum.

Sequencing of fli $\mathrm{C}_{E A E C}$ and comparison to other bacterial flagella. The nucleotide sequence of fli $C_{\mathrm{EAEC}}$ was obtained from pfliC (submitted to GenBank; accession AF 194946). The predicted amino acid sequence of this gene is shown in comparison to flagellins from S. dysenteriae, S. flexneri, and E. coli K12 in Figure 7. The four proteins are nearly identical over the first 150 and last 90 amino acids. FliC-EAEC and FliC-SD are 


\section{Figure 7}

Comparison of deduced amino acid sequences of flagellins from EAEC 042 (EAEC), S. dysenteriae (SD), S. flexneri (SF), and E. coli K12 (K12). Trypsin peptides identified by mass spectrometry from the initial purification product of EAEC 042 supernatants are underlined. Amino acids in the EAEC flagellin differing from those in $S$. dysenteriae are shown above the line. The arrows represent the locations of the forward and reverse primers used to generate the 588-bp fliC probe. FliC-EAEC accession AF 194946.
1

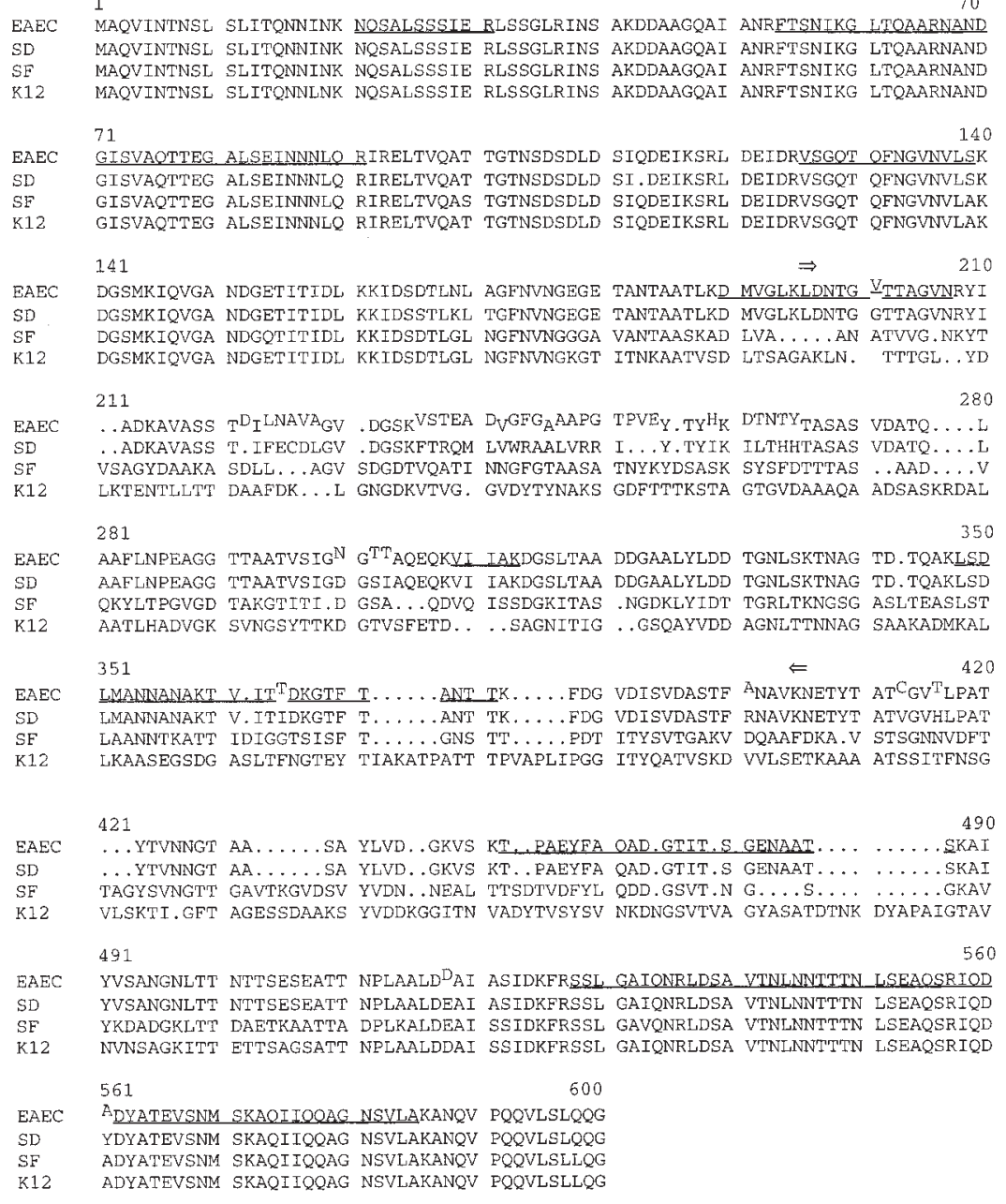
40 OENGVNVLSK SE GISVAOTTEG ALSEINNNEQ RIRELTVQAS TGTNSDSDLD SIODEIKSRL DEIDRVSGOT QFNGVNVLAK K12 GISVAQTTEG ALSEINNNLQ RIRELTVQAT TGTNSDSDLD SIODEIKSRL DEIDRVSGQT OFNGVNVLAK

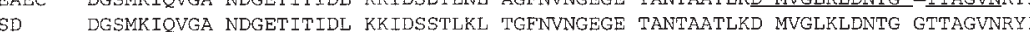
DGSMKIOVGA NDGOTITTDL KKIDSDTLGL NGFNVNGGGA VANTAASKAD LVA.....AN ATVVG.NKYT GSMKIOVGA NDGETITIDL KKIDSDTLGL NGFNVNGKGT ITNKAATVSD LTSAGAKLN. TTTGL. YD D.

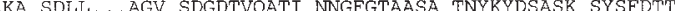

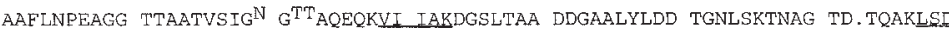
AAFLNPEAGG TTAATVSIGD GSIAOEOKVI IAKDGSLTAA DDGAALYLDD TGNLSKTNAG TD.TOAKLSD THEV TAKGTITI.D GSA...QDVQ ISSDGKITAS .NGDKLYIDT TGRLTKNGSG ASLTEASLST LMANNANAKT $V$. ITIDKGTE T .... ANT TKK ....FDG VDISVDASTE RNAVKNETYT ATVGVHLPAT A.ANNTKATT IDIGGTSISE T......GNS TT...... PDT ITYSVTGAKV DQA.AFDKA.V STSGAINVDFT KAASEGSDG ASLTFNGTEY TIAKATPATT TPVAPLIPGG ITYQATVSKD VVLSETKAAA ATSSITFNSG .......SKAI TINK DYAPAIGTAV 560 YVSANGNLTT NITSESEATT NPLAALDEAI ASIDKERSSL GAIQNRLDSA VTNLNNTTTN LSEAQSRIQD YKDADGKLTT DAETKAATTA DPLKALDEAI SSIDKFRSSL GAVQNRLDSA VTNLNNTTTN LSEAQSRIQD
NVNSAGKITT ETTSAGSATT NPLAALDDAI SSIDKERSSL GAIQNRLDSA VT'NLNN'TITN LSEAQSRIQD completely identical over the first 200 residues, and more than $98 \%$ identical over the last 420 , but only $42 \%$ identical from amino acid 220 to 270 . A database search of this region of FliC-EAEC using the FASTA program from Genetics Computer Group (Madison, Wisconsin, USA) found no significant homology to any known protein.

\section{Discussion}

We have demonstrated that the IL-8-releasing activity of EAEC 042 can be attributed to a single protein moiety that has a sequence very similar to that of a flagellin from S. dysenteriae. Furthermore, we have shown that flagella from 042 and several other EAEC isolates are extremely potent in releasing IL-8, and that aflagellar 042 loses its IL-8-releasing activity. Finally, EAEC flagellin expressed as a His-tagged fusion protein possesses the same activity. These findings show that the EAEC IL-8-releasing factor is in fact a flagellar protein that interacts with epithelial cells, leading to IL-8 secretion.

Interestingly, whereas flagella have been implicated in the production of inflammation by several other gram-negative pathogens, this activity has not previously been attributed to a single gene product.
Pseudomonas aeruginosa aflagellar mutants demonstrate reduced pathogenicity in the mouse pneumonia model, and purified flagella can themselves produce pulmonary inflammation (12). In addition, $P$. aeruginosa isolates with altered flagellar expression demonstrate reduced ability to release IL-8 from A549 pulmonary epithelial cells (13). Helicobacter pylori isolates with natural polymorphisms in flagellar genes induce different amounts of IL-8 release from MKN45 gastric epithelial cells, and IL-8 release correlates with motility (14). Purified phase I Salmonella flagellin and Salmonella fliC expressed in E. coli each cause release of TNF- $\alpha$ from a human promonocytic cell line (15). Finally, Salmonella typhi flagella impair antigen uptake and presentation by human macrophages (16), and induce a cytokine cascade in these cells (17).

Whereas most E. coli express flagella and are motile, the closely related Shigella species were until recently thought to be devoid of flagella and constitutively nonmotile. However, Tominaga et al. demonstrated that $S$. flexneri and S. sonnei both possess genes homologous to fliC that can be expressed in E. coli to produce flagella (18). A corresponding sequence for $S$. dysenteriae was 
submitted to GenBank. However, this putative protein has not been described in a publication, nor has any function been ascribed to it.

Flagellar operons are present in all four Shigella subgroups, but all contain disrupting elements that prevent coordinated expression of intact flagella (19, 20). Nevertheless, Girón demonstrated that under carefully controlled conditions, Shigella can in fact be induced to express flagella, but in very low numbers (one flagellum per $300-1,000$ organisms), and that they do exhibit some motility in very soft $(0.175 \%)$ agar (21). The high degree of homology between the flagellin from EAEC 042 and that from S. dysenteriae and $S$. flexneri raises the intriguing hypothesis that expression of flagellin in vivo may contribute to the severe inflammation characteristic of shigellosis.

A comparison of the predicted amino acid sequence of FliC-EAEC with that of other flagellins reveals substantial homology at the $\mathrm{NH}_{2}$ and $\mathrm{COOH}$ termini. This is consistent with the model of flagellin structure proposed by Vonderviszt et al. (22). In monomeric form, flagellin consists of disordered $\mathrm{NH}_{2}$ - and $\mathrm{COOH}$-terminal regions and three organized domains, labeled G1, G2, and G3. The G3 domain, formed by the $\mathrm{NH}_{2}$ - and $\mathrm{COOH}$-subterminal regions of the molecule, forms a compact structure and is highly conserved among many E. coli flagellins (23); it presumably is required for proper assembly of the molecule. In contrast, the G1 and G2 domains, which are formed by the middle of the molecule, are hypervariable and presumed to contain the $\mathrm{H}$-antigenic epitopes. The divergence in G1 and G2 domains has been proposed to be driven by antigenic diversity (as a way to avert host response); our work suggests that these domains may confer important and heretofore unrecognized pathogenic phenotypes.

The expression of proinflammatory flagella by EAEC may be an important and novel pathogenic characteristic of these organisms. The phenotype of aggregative adherence, which is pathognomonic of EAEC, is one of several virulence traits identified in these organisms, but is not associated with inflammation. Along with aggregative adherence fimbriae, the large AA plasmid in some isolates carries the genes for Pet, a $104-\mathrm{kDa}$ serine protease enterotoxin and cytotoxin; EAST-1, a small ST-like polypeptide enterotoxin; and several cryptic loci with homology to virulence genes of other bacterial species. None of these genes is required for IL- 8 release; plasmid-cured 042 maintains its activity (8). Virulence traits present on the EAEC 042 chromosome include pic mucinase, which is encoded on the antisense strand in an overlapping configuration with Shigella enterotoxin 1 (24), and putative genes for yersiniabactin (25) and a putative invasion protein, Tia (26). Although these genes may confer pathogenic phenotypes in vivo, there is no evidence that they are involved in producing inflammation.

Proinflammatory flagella could play an important role in EAEC pathogenesis by inducing epithelial IL-
8 production and recruiting neutrophils to the epithelial surface. In other intestinal infections, neutrophil transmigration has been shown to cause fluid secretion and epithelial barrier disruption $(27,28)$. This could enhance the effects of other EAEC toxins such as Pet or EAST-1, although whether this occurs in vivo remains to be seen. The presence of IL8-releasing flagella in diverse EAEC isolates with heterogeneity in other pathogenic genotypes suggests that these flagella may be pathogenic independent of other EAEC toxins. Further studies will be needed to determine the range of effects of proinflammatory flagella on the intestinal epithelium. These findings would in turn lead to a better understanding of how EAEC and other intestinal bacteria produce persistent diarrhea, intestinal inflammation, growth impairment, and other potentially unrecognized manifestations of early childhood infection.

\section{Acknowledgments}

We would like to acknowledge the invaluable advice and assistance of Robert Nakamoto, Joanna Goldberg, Barbara Mann, and Chang-Hun Yang. This work was supported by National Institutes of Health grants to R.L. Guerrant (99/00.UFB.4 and AI-26512), J.P. Nataro (AI-33096), and T.S. Steiner (AI-01573), the National Foundation for Infectious Diseases-Glaxo Wellcome Fellowship in Infectious Diseases, and the Burroughs Wellcome Fund Career Award in the Biomedical Sciences, to T.S. Steiner.

1. Nataro, J., Steiner, T., and Guerrant, R. 1998. Enteroaggregative Escherichia coli. Emerg. Infect. Dis. 4:251-261.

2. Nataro, J., et al. 1987. Patterns of adherence of diarrheagenic Escherichia coli to HEp-2 cells. Pediatr. Infect. Dis. J. 6:829-831.

3. Cohen, M.B., et al. 1993. Colonization by enteroaggregative Escherichia coli in travelers with and without diarrhea. J. Clin. Microbiol. 31:351-353.

4. Gascon, J., et al. 1998. Enteroaggregative Escherichia coli strains as a cause of traveler's diarrhea: a case-control study. J. Infect. Dis. 177:1409-1412.

5. Mayer, H.B., and Wanke, C.A. 1995. Enteroaggregative Escherichia coli as a possible cause of diarrhea in an HIV-infected patient. N. Engl. J. Med. 332:273-274.

6. Oberhelman, R., et al. 1997. Enteroadherent, enterotoxigenic, and enterohemorrhagic $E$. coli infections in day care center attendees in New Orleans. 46th Annual Meeting of the American Society of Tropical Medicine and Hygiene. Lake Buena Vista, Florida, USA. p. 308.

7. Itoh, Y., Nagano, I., Kunishima, M., and Ezaki, T. 1997. Laboratory investigation of enteroaggregative Escherichia coli $\mathrm{O}$ untypable:H10 associated with a massive outbreak of gastrointestinal illness. J. Clin. Microbiol. 35:2546-2550.

8. Steiner, T., Lima, A., Nataro, J., and Guerrant, R. 1998. Enteroaggregative Escherichia coli produce intestinal inflammation and growth impairment and cause interleukin- 8 release from intestinal epithelial cells. J. Infect. Dis. 177:88-96.

9. Guerrant, D.I., et al. 1999. Association of early childhood diarrhea and cryptosporidiosis with impaired physical fitness and cognitive function four-seven years later in a poor urban community in northeast Brazil. Am. J. Trop. Med. Hyg. 61:707-713.

10. Nataro, J., et al. 1995. Heterogeneity of enteroaggregative Escherichia coli virulence demonstrated in volunteers. J. Infect. Dis. 171:465-468.

11. Penfold, R., and Pemberton, J. 1992. An improved suicide vector for construction of chromosomal insertion mutations in bacteria. Gene. 118:145-146.

12. Feldman, M., et al. 1998. Role of flagella in pathogenesis of Pseudomonas aeruginosa pulmonary infection. Infect. Immun. 66:43-51.

13. DiMango, E., Zar, H.J., Bryan, R., and Prince, A. 1995. Diverse Psendomonas aeruginosa gene products stimulate respiratory epithelial cells to produce interleukin-8. J. Clin. Invest. 96:2204-2210.

14. Ohta-Tada, U., Takagi, A., Koga, Y., Kamiya, S., and Miwa, T. 1997. Fla- 
gellin gene diversity among Helicobacter pylori strains and IL-8 secretion from gastric epithelial cells. Scand. J. Gastroenterol. 32:455-459.

15. Ciacci-Woolwine, F., Blomfield, I.C., Richardson, S.H., and Mizel, S.B. 1998. Salmonella flagellin induces tumor necrosis factor alpha in a human promonocytic cell line. Infect. Immun. 66:1127-1134.

16. Wyant, T.L., Tanner, M.K., and Sztein, M.B. 1999. Potent immunoregulatory effects of Salmonella typhi flagella on antigenic stimulation of human peripheral blood mononuclear cells. Infect. Immun. 67:1338-1346.

17. Wyant, T.L., Tanner, M.K., and Sztein, M.B. 1999. Salmonella typhi flagella are potent inducers of proinflammatory cytokines. Infect. Immun. 67:3619-3624.

18. Tominaga, A., Mahmoud, M.A., Mukaihara, T., and Enomoto, M. 1994. Molecular characterization of intact, but cryptic, flagellin genes in the genus Shigella. Mol. Microbiol. 12:277-285.

19. Al Mamun, A.A., Tominaga, A., and Enomoto, M. 1996. Detection and characterization of the flagellar master operon in the four Shigella subgroups. J. Bacteriol. 178:3722-3726.

20. Al Mamun, A.A., Tominaga, A., and Enomoto, M. 1997. Cloning and characterization of the region III flagellar operons of the four Shigella subgroups: genetic defects that cause loss of flagella of Shigella boydi and Shigella sonnei. J. Bacteriol. 179:4493-4500.

21. Girón, J.A. 1995. Expression of flagella and motility by Shigella. Mol. Microbiol. 18:63-75.

22. Vonderviszt, F., Uedaira, H., Kidikoro, S., and Namba, K. 1990. Structural organization of flagellin. J. Mol. Biol. 214:97-104.
23. Reid, S.D., Selander, R.K., and Whittam, T.S. 1999. Sequence diversity of flagellin (fliC) alleles in pathogenic Escherichia coli. J. Bacteriol. 181:153-160.

24. Henderson, I.R., Czeczulin, J., Eslava, C., Noriega, F., and Nataro, J.P. 1999. Characterization of Pic, a secreted protease of Shigella flexneri and enteroaggregative Escherichia coli. Infect. Immun. 67:5587-5596.

25. Schubert, S., Rakin, A., Karch, H., Carniel, E., and Heeseman, J. 1998. Prevalence of the "high-pathogenicity island" of Yersinia species among Escherichia coli strains that are pathogenic to humans. Infect. Immun. 66:480-485.

26. Fleckenstein, J.M., Kopecko, D.J., Warren, R.L., and Elsinghorst, E.A. 1996. Molecular characterization of the tia invasion locus from enterotoxigenic Escherichia coli. Infect. Immun. 64:2256-2265.

27. Madara, J.L., Patapoff, T.W., and Gillece-Castro, B. 1993. Adenosine monophosphate is the neutrophil-derived paracrine factor that elicits chloride secretion from T84 intestinal epithelial cells. J. Clin. Invest. 91:2320-2325.

28. Sansonetti, P.J., Arondel, J., Huerre, M., Harada, A., and Matsushima, K. 1999. Interleukin-8 controls bacterial transepithelial translocation at the cost of epithelial destruction in experimental shigellosis. Infect. Immun. 67:1471-1480.

29. Kaper, J.B., Lockman, H., Baldini, M.M., and Levine, M.M. 1984. Recombinant nontoxigenic Vibrio cholerae strains as attenuated cholera vaccine candidates. Nature. 308:655-658.

30. Donnenberg, M.S., and Nataro, J.P. 1995. Methods for studying adhesion of diarrheagenic Escherichia coli. Methods Enzymol. 253:324-336. 